\title{
Cloning and characterization of a novel sigma-like glutathione S-transferase from the giant panda parasitic nematode, Baylisascaris schroederi
}

Yue Xie ${ }^{1+}$, Xuan Zhou ${ }^{2+}$, Lin Chen', Zhihe Zhang ${ }^{3}$, Chengdong Wang ${ }^{4}$, Xiaobin Gu', Tao Wang ${ }^{1}$, Xuerong Peng ${ }^{5}$ and Guangyou Yang ${ }^{1 *}$

\begin{abstract}
Background: Baylisascaris schroederi, an intestinal nematode of the giant panda, is the cause of the often fatal disease, baylisascariasis. Glutathione S-transferases (GSTs) are versatile enzymes that can affect parasite survival and parasite-host interactions and, are therefore, potential targets for the development of diagnostic tests and vaccines.

Methods: In this study, we identified a full-length CDNA that encoded a novel, secretory sigma-like GST (BSC-GSTб) from a B. schroederi-omic dataset. Following cloning and sequencing, sequence and structural analyses and comparative

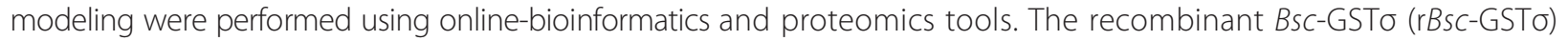
protein was prokaryotically expressed and then used to detect antigenicity and reactivity using immunoblotting assays. In addition, the native protein in female adult B. schroederi was located via immunofluorescence techniques, while the preliminary ELISA-based serodiagnostic potential of rBsc-GSTo was assessed in native and infected mouse sera.

Results: Bsc-GSTo contained a 621-bp open reading frame that encoded a polypeptide of 206 amino acids with two typical sigma GST domain profiles, including a GST_N_Sigma_like at the N-terminus and a GST_C_Sigma_like at the $\mathrm{C}$-terminus. The presence of an $\mathrm{N}$-terminal signal sequence indicated that BsC-GSTo was a secretory protein. Sequence alignment and phylogenetic analyses showed that Bsc-GSTo was a nematode-specific member of the Sigma class GSTs and shared the closest genetic distance with its homologue in Ascaris suum. Further comparative structure analyses indicated that Bsc-GSTo possessed the essential structural motifs (e.g., $\beta a \beta a \beta \beta a$ ) and the consensus secondary or tertiary structure that is typical for other characterized GSTos. Immunolocalization revealed strong distributions of native Bsc-GSTo in the body hypodermis, lateral chords, gut epithelium, gut microvilli, oviduct epithelium, and ovaries of adult female worms, similar to its homologue in A. suum. Building on good immunogenic properties, rBsc-GSTo-based ELISA exhibited a sensitivity of $79.1 \%$ and a specificity of $82.0 \%$ to detect anti-B. schroederi lgG antibodies in the sera of experimentally infected mice.

Conclusion: This study presents a comprehensive demonstration of sequence and structural-based analysis of a new, secretory sigma-like GST from a nematode, and its good serodiagnostic performance suggests that rBsc-GSTo has the potential to detect $B$. schroederi and, therefore, could be used to develop an ELISA-based serological test to diagnose baylisascariasis in giant pandas.
\end{abstract}

Keywords: Baylisascaris schroederi, Giant panda, Glutathione S-transferase, Sigma class, Bsc-GSTo, Immunolocalization, Immunodiagnosis

\footnotetext{
* Correspondence: guangyou1963@aliyun.com

${ }^{+}$Equal contributors

${ }^{1}$ Department of Parasitology, College of Veterinary Medicine, Sichuan

Agricultural University, Ya'an 625014, China

Full list of author information is available at the end of the article
}

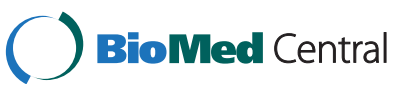

(c) 2015 Xie et al.; licensee BioMed Central. This is an Open Access article distributed under the terms of the Creative Commons Attribution License (http://creativecommons.org/licenses/by/4.0), which permits unrestricted use, distribution, and reproduction in any medium, provided the original work is properly credited. The Creative Commons Public Domain Dedication waiver (http://creativecommons.org/publicdomain/zero/1.0/) applies to the data made available in this article, unless otherwise stated. 


\section{Background}

The giant panda (Ailuropoda melanoleuca) is one of the world's most iconic and endangered species, and is currently confined to several small mountain habitats of western China (Qinling, Minshan, Qionglai, Daxiangling, Xiaoxiangling, and Liangshan) with a population size of $\sim 1600[1,2]$. Wild giant pandas face the threat of extinction from human population expansion, destruction of their habitat, and the detrimental impacts of parasites and other diseases $[3,4]$. Baylisascariasis, caused by the nematode, Baylisascaris schroederi (Nematoda: Ascaridida), is one of the leading causes of death for both wild and captive giant pandas and has been responsible for half of the recorded deaths of pandas from 2001 to 2005 $[3,5]$. As with other ascaridoids, adult B. schroederi usually inhabit the intestines of the giant panda, while the larvae may disseminate into various body tissues. In pandas, damage to bodily tissues includes extensive inflammation and scarring of the intestinal wall and parenchyma of the liver and lung (also known as visceral larval migran, VLM; caused by larvae), as well as intestinal obstruction, inflammation, and even death (caused by adults) [5-8]. Currently, diagnosis and identification of $B$. schroederi infection in pandas relies on morphological examination of fecal eggs, which requires extensive expertise and is difficult, laborious, and prone to error (as the density of eggs in bamboos-enriched feces is low and subject to possible environmental crosscontaminating with the eggs of other parasites, including morphologically similar Baylisascaris spp. [5]). Recently, a new molecular method to detect $B$. schroederi was developed based on the PCR-based detection of mitochondrial makers (COII or 12S) [9-11]. This method, however, cannot diagnose migrating larvae or adults outside of the egglaying period. Hence, an alternative and more efficient molecular tool is needed. Serodiagnosis, particularly the ELISA tests (enzyme-linked immunosorbent assays) equipped with target molecules that play excretory/ secretory (ES) roles and function in the survival, development, and immune evasion of parasites [12], would be an ideal and better strategy due to its sensitivity and clinical practices.

Glutathione S-transferases (GSTs; EC 2.5.1.18) are a versatile protein superfamily that are widely distributed among all living cells and act in cellular detoxification and protection via either catalyzing toxin conjugation with reduced glutathione $(\mathrm{GSH})$ or passively binding to various exogenous/endogenous toxic molecules, including carcinogens, therapeutic agents, and products of oxidative stress $[13,14]$. For parasites, some secretory GSTs are further believed to be associated with parasite survival, repair of damage caused by host's immune-initiated reactive oxygen species (ROS), transportation or metabolism of essential materials, and host immune modulation
[12,14-19]. Encouragingly, because of these important functions, some parasite-derived GSTs, including those of parasitic nematodes, have been selectively targeted for vaccine development and diagnosis purposes [15,16,19-23]. For example, a secretory sigma-class GST from Ascaris lumbricoides (GSTA) has recently been identified and investigated as a new allergen for clinical diagnosis of the human roundworm disease [24], although the frequency of the antibody (mainly IgE) sensitization to GSTA is not high and the GSTA exhibits several isoforms with differential IgE recognition. Also, another secretory GST-3 from the human filarial nematode Onchocerca volvulus (OvGST3) is under investigation as a potential antigen candidate for the diagnosis of onchocerciasis due to its high exposure to the human host's immune system and good immunogenic properties [19].

Given that most recently described nematode-derived GSTs are from the Sigma class in term of their sequence homology, structure, substrate specificity, immunological and phylogenetic analyses [20,22], and that no information on GSTs of B. schroederi is available to date; importantly, B. schroederi-specific immunogenic proteins as potential diagnostic agents are lacking, herein, the aims of this study were to (i) clone and express a new, secretory sigma-like GST, Bsc-GST $\sigma$, from B. schroederi; (ii) characterize its potential functions by locating the native protein in adult parasites; and (iii) test the immunogenicity and preliminary ELISA-based diagnostic potential of its recombinant Bsc-GST ( $\mathrm{r} B s c-\mathrm{GST} \sigma)$ in mice using native and infected sera. The results of this work will provide the foundation for the further development of Bsc-GST as a candidate serodiagnostic antigen to detect B. schroederi in the giant panda.

\section{Methods}

\section{Ethics statement}

This study was reviewed and approved by the Animal Ethics Committee of Sichuan Agricultural University (AECSCAU; Approval No. 2011-028). Animals were handled strictly accordance with the animal protection law of the People's Republic of China (released on 09/18/2009) and the National Standards for Laboratory Animals in China (executed on 05/1/2002).

\section{Animals}

Female specific-pathogen-free (SPF) BALB/c mice (6-8 weeks old) were purchased from the Laboratory Animal Center of Sichuan University (Chengdu, China). New Zealand white rabbits were obtained from the Laboratory Animal Center of Sichuan Agricultural University (Ya'an, China). All animals were housed under a barrier environment in sterile cages and provided with pelleted food and sterilized water ad libitum. Animals were acclimated to these conditions for one week prior to the experiment. 


\section{Parasites}

B. schroederi female adults derived from naturally infected giant pandas were provided by the Department of Parasitology, College of Veterinary Medicine, Sichuan Agricultural University. Adult female Ascaris suum and Baylisascaris transfuga were isolated from infected pigs at a local slaughterhouse in Ya'an and an infected polar bear after treatment with pyrantel pamoate in Chengdu zoological garden, China, respectively. Embryonated and/ or un-embryonated eggs were obtained from the respective dissections of the uteruses of B. schroederi, B. transfuga, and A. suum using established procedures [25]. The infective egg-L2 larvae of these three ascaridoids were collected from subsequent incubation of the embryonated eggs according to the USEPA and Tulane methods [26,27]. All L2-contained eggs were stored at $4{ }^{\circ} \mathrm{C}$ until use.

\section{RNA isolation, amplification and bioinformatic analysis of Bsc-GSTo-1}

Total RNA was isolated from an adult female specimen of B. schroederi using an RNA extraction kit (Clontech, Palo Alto, CA) according to the manufacturer's directions. The isolated RNA was subsequently subjected to first-strand cDNA synthesis using a cDNA synthesis kit and an oligo $(\mathrm{dT})_{18}$ primer (MBI Fermentas, Germany). The resulting double-stranded $\mathrm{cDNA}$ was used as the template for PCR amplification with the sense primer (5'-AAG CAACATGCCGCAGTACAAG-3') and the antisense primer (5' -CACAAAAAACAGAATAGACCCTAATA-3') designed to target a full-length coding sequence of the GST homologue that was screened from the assembled and annotated B. schroederi genome (Scaffold ID 47) and transcriptome (Unigene ID 86248) datasets (data unpublished). The amplified product was gel-purified, cloned into the pMD19-T vector (TaKaRa, Dalian, China) and then sequenced. After a homology search by BLASTn (http://blast.ncbi. nlm.nih.gov/Blast.cgi?PROGRAM=blastn\&PAGE_TY$\mathrm{PE}=$ BlastSearch\&LINK_LOC=blasthome), the cDNA showed similarity to known sigma GSTs and therefore was designated as Bsc-GSTo. The open reading frame (ORF) and deduced amino acid sequence of Bsc-GSTo were derived using an Open Reading Frame Finder and the Lasergene software package for Windows (DNASTAR, Madison, WI, USA) and then assessed with ExPASy online servers (http:// www.expasy.org/). Conserved domains (CD) were identified using the CD-Database-based PROSITE profile analysis (http://www.ncbi.nlm.nih.gov/Structure/cdd/cdd.shtml). The isoelectric point (pI) and molecular weight $(\mathrm{Mw})$ of Bsc-GST $\sigma$ were calculated using the Compute $\mathrm{pI} / \mathrm{Mw}$ tool (http://web.expasy. org/compute_pi/) and the signal sequence was predicted with the SignalP 4.0 server (http://www.cbs.
dtu.dk/services/SignalP/). Similarity comparisons with previously reported sequences were conducted using DNAMAN 3.0 (Lynnon Biosoft, Quebec, Canada) and online BLASTp tool (http://blast.ncbi.nlm.nih. gov/Blast.cgi?PROGRAM=blastp\&PAGE_TYPE=BlastSearch\&LINK_LOC=blasthome). On the basis of observed similarities, a multiple sequence alignment and phylogenetic analysis were conducted. Sequences were aligned with ClustalW2 and the phylogenetic tree was constructed using the neighbor-joining (NJ) method [28] and plotted with MEGA 5.0 [29]. In addition, for structural modeling of Bsc-GST $\sigma$, we used the YASPIN secondary structure prediction program (http://www.ibi.vu. $\mathrm{nl} /$ programs/yaspinwww/) to infer secondary structure. Tertiary (3D) structure was assessed through the CPHmodels3.2 Server online program (http://www.cbs.dtu.dk/services/ CPHmodels/) and by referring to the $1.90 \AA$ resolution crystal structure of $N$. americanus GSTo2 (PDB accession no.: 2ON5).

\section{Expression and purification of $\mathrm{rBsc}-\mathrm{GST} \sigma$}

Due to the presence of the predicted signal peptide, a region encoding mature Bsc-GST $\sigma$ was amplified by PCR for expression using the following primers: $5^{\prime}$-CCCGGATC CATTCGTGGCCTGGGTG-3' (forward; a BamHI site in italics) and 5'-CGCAAGCTTCACAAAAGCAGAAGAGA CTCTAATA-3' (reverse; a HindIII site in italics). After enzyme digestion with BamHI and HindIII (TaKaRa) and gelpurification, this fragment was ligated into the plasmid expression vector pET32a (+) (Novagen, Madison, USA). The correct resulting plasmid was confirmed by sequencing, transformed into E. coli BL21 (DE3) cells (Invitrogen, Carlsbad, USA), and then grown in Luria-Bertani (LB) broth with $100 \mathrm{mg} / \mathrm{mL}$ of ampicillin at $37^{\circ} \mathrm{C}$ until the optical density at $600 \mathrm{~nm}$ reached 0.6. Expression was induced by adding $1 \mathrm{mM}$ isopropyl $\beta$-D-thiogalactopyranoside (IPTG) for an additional $4 \mathrm{~h}$ culture at $37^{\circ} \mathrm{C}$. The cells were pelleted and suspended in lysis buffer $\left[50 \mathrm{mM} \mathrm{NaH} \mathrm{PO}_{4}\right.$ (pH 8.0), $10 \mathrm{mM}$ Tris- $\mathrm{HCl}$ (pH 8.0), $100 \mathrm{mM} \mathrm{NaCl}$. The samples were subjected to sonication and centrifuging to enable collection of inclusion bodies and cellular debris and the removal of other soluble substances. His ${ }_{6}$-tagged recombinant Bsc-GSTo proteins were expressed as inclusion bodies in the pellets after sodium dodecyl sulfatepolyacrylamide gel electrophoresis (SDS-PAGE) and then purified using $\mathrm{Ni}^{2+}$ affinity chromatography (Novagen) under denaturing conditions according to the manufacturer's protocol. Refolding of the purified recombinant proteins was performed as recommended elsewhere [8]. Thereafter, the refolded protein was concentrated using the vacuum freeze-drying technique and its concentration was determined with the micro-bicinchoninic acid protein assay reagent (Pierce/Thermo Fisher Scientific, Asheville, USA). 
Potential contamination by endotoxins was assessed using the limulus amoebocyte lysate-based gel-clot assay [30].

\section{Sera}

Mouse immune sera against parasites A. suum (15 samples), B. transfuga (15 samples) or B. schroederi (43 samples) were produced for serodiagnostic assays as previously described $[8,31,32]$. Rabbit anti-B. schroederi sera were generated as follows: 3,600 B. schroederi infective embryonated eggs were administered to one New Zealand white rabbit, followed by four repeat inoculations every 2 weeks. The rabbit was bled two weeks after the final inoculation and the serum was collected for immunoblotting analysis. To obtain mouse polyclonal sera against $\mathrm{rBsc}-\mathrm{GST} \sigma, 10 \mathrm{BALB} / \mathrm{c}$ mice were subcutaneously immunized with $50 \mu \mathrm{g}$ of purified $\mathrm{rBsc}$ GSTo mixed with FCA (Sigma, St. Louis, USA), followed by two booster immunizations (two weeks apart) using the same route and dose in the same adjuvant. Mice were bled two weeks after the final immunization. The anti$\mathrm{rBsc-GST} \sigma$ sera were mixed and stored at $-20^{\circ} \mathrm{C}$ until use. Additionally, 20 mouse native sera were provided by the Department of Parasitology, College of Veterinary Medicine, Sichuan Agricultural University.

\section{Immunoblotting and immunolocalization}

For immunoblotting analysis, $\mathrm{rBsc}-\mathrm{GST} \sigma$ was lysed in an electrophoresis sample buffer, run on 10\% SDS-PAGE and subsequently transferred onto the nitrocellulose membrane. The blotting membrane was incubated with 5\% skim milk in Tris-buffered saline (TBS) buffer for $1 \mathrm{~h}$. To better test the antigenicity of $\mathrm{rBsc}-\mathrm{GST} \sigma$, rabbit immune sera from animals repeatedly inoculated with $B$. schroederi embryonated infective eggs, anti-rBsc-GST $\sigma$ mice serum, and native rabbit or mouse serum were included here. After the membrane was washed three times with TBSTween 20 (TBST), it was further incubated for $2 \mathrm{~h}$ with 1:200 diluted alkaline phosphatase-conjugated goat antimouse or anti-rabbit IgG (ICN Pharmaceuticals, Costa Mesa, CA). Following the same washing steps described above, the protein signals were visualized using nitroblue tetrazolium and 5-bromo-4-chloro-3-in-dolylphosphate (NBT/BCIP; USB, Cleveland, OH) and recorded using Image Quant LAS-4000 (GE Healthcare Life Sciences, USA). For immunolocalization studies, adult female B. schroederi sections were probed with specific mouse anti-rBsc-GSTo serum (1:100) and then with fluorescein isothiocyanate (FITC)-labeled goat anti-mouse IgG (1:100; Santa Cruz, CA, USA) as described elsewhere [33]. The stained samples were mounted in glycerol/phosphate buffer (v/v, 9:1) and viewed under an Olympus BX50 fluorescence microscope (Olympus, Japan).

\section{ELISA}

To assess the preliminary serodiagnostic potential of $\mathrm{rBsc-GST \sigma}$, B. schroederi-specific IgG antibodies of mice were detected by ELISA following the methodology described by Vlaminck et al. [34], with some modifications. In brief, ELISAs were performed in polystyrene 96-well microtiter plates (Invitrogen) using $100 \mu \mathrm{L}$ reaction mixtures with $\mathrm{rBsc}$-GST $\sigma$ antigen coated at three different concentrations $(1,2$, and $4 \mu \mathrm{g} / \mathrm{mL})$ in $0.1 \mathrm{M}$ carbonate buffer $\left(\mathrm{pH}\right.$ 9.6). After overnight incubation at $4^{\circ} \mathrm{C}$, all plates were washed with PBS $+0.5 \%$ Tween 20 (PBST20) and then the wells were blocked with $100 \mu \mathrm{L}$ of PBS-2\% bovine serum albumin (BSA) for $2 \mathrm{~h}$ at $37^{\circ} \mathrm{C}$. A serial two-fold dilutions (100 $\mu \mathrm{L}$; ranging from $1: 100$ to $1: 1600)$ of the positive serum sample (provided by our lab) and goat anti-mouse IgG-HRP conjugate (100 $\mu \mathrm{L} ; 1: 500)$ (Boster Bio-project Co., Wuhan, China) were used in the following steps, and positive sera and conjugates were diluted in PBS. Antibody binding was detected with $100 \mu \mathrm{L}$ of $\mathrm{O}$-phenylenediamine dihydrochloride substrate $(0.4 \mathrm{mg} / \mathrm{mL}$ OPD, $50 \mathrm{mM}$ dibasic sodiumphosphate, $25 \mathrm{mM}$ citric acid, and $30 \% \mathrm{H}_{2} \mathrm{O}_{2}$ ), and the optical density (OD) was measured at $492 \mathrm{~nm}$. Negative and blank controls were included on each plate. After the best dilutions of $\mathrm{rBsc}-\mathrm{GST} \sigma$ antigen and mouse serum were determined, 93 mouse serum samples (43 for B. schroederi-positive, 30 for A. suum/B. transfugapositive, and 20 for control) were serodiagnosed with the ELISA. The sensitivity of the assay was calculated as follows: sensitivity $(\%)=$ ELISA positive/true positive $\times 100$, while the specificity was evaluated by the cross-reaction with the heterosera derived from mice infection with the congeneric species, $B$. transfuga, and the related ascaridoid, A. suum.

\section{Statistical analysis}

ELISA data were expressed as the mean value \pm standard deviation (SD). Comparisons between test sera groups were carried out using one-way ANOVA in SPSS version 17.0 for Windows (SPSS Inc., Chicago, IL). $P$ values $<0.05$ were considered to be significant. The negative cut-off was calculated as the mean value $+3 \times$ SD from the OD values of the normal sera, as previously described [35]. Additionally, the nucleotide sequence determined in the present study was deposited in the GenBank database under accession number KM435257.

\section{Results}

Molecular characterization of Bsc-GSTo

The novel sigma-like GST, Bsc-GST $\sigma$, was initially identified through homologously screening the assembled and annotated $B$. schroederi genome and transcriptome datasets. The full-length Bsc-GST $\sigma$ gene sequence within the genomic DNA was 4,340 bp in size and comprised 


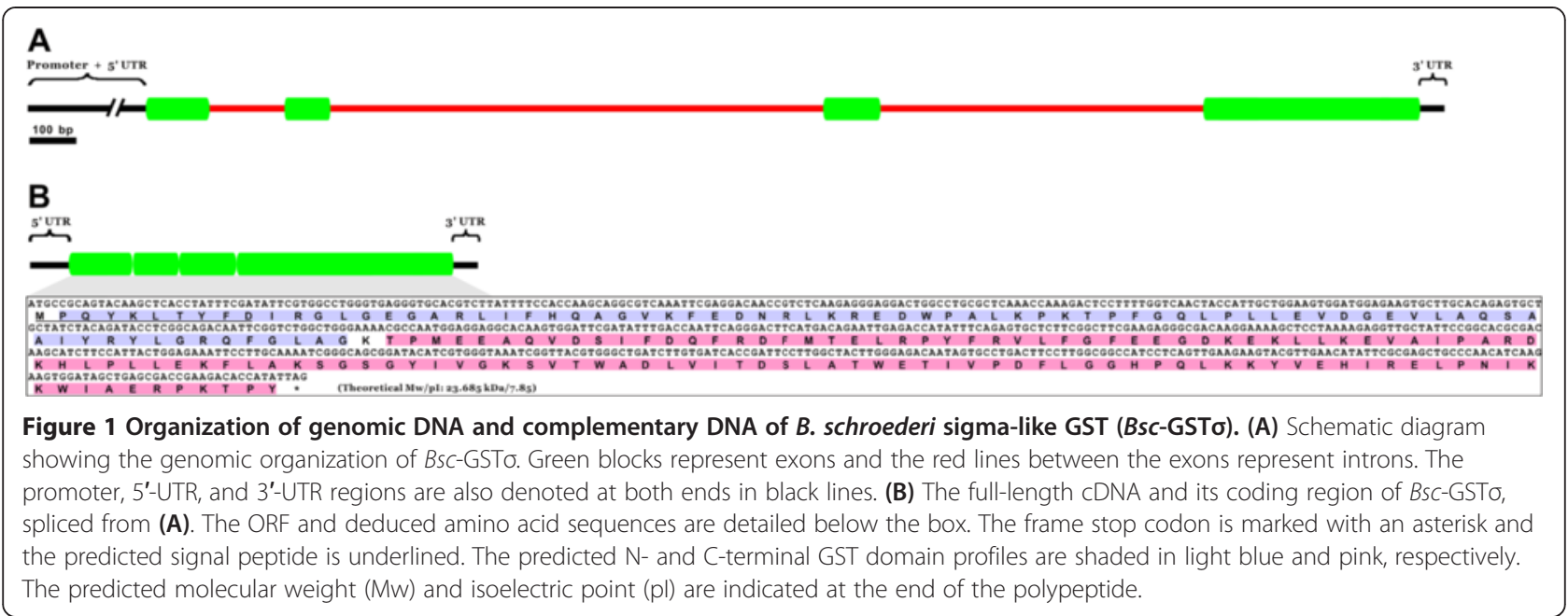

four exons $(95,124,133$, and $269 \mathrm{bp})$ and three introns $(160,386$, and $546 \mathrm{bp})$ (Figure 1A). The nucleotide sequence at the splice junctions is consistent with the canonical GT-AG rule [36]. The full-length cDNA of $B s c-G S T \sigma$ was 725 bp in size and contained a single ORF of $621 \mathrm{bp}$, a 5 'untranslated region (UTR) of $30 \mathrm{bp}$, and a 3' UTR of 74 bp (Figure 1B). The complete ORF of Bsc-GSTo isolated here encoded a polypeptide of 206 amino acids with a predicted $\mathrm{Mw}$ of $23.685 \mathrm{kDa}$ and a $\mathrm{pI}$ of 7.85. The PROSITE domain analysis revealed that the deduced Bsc-GSTo polypeptide had two typical GST domain profiles at the $\mathrm{N}-(2-79$ amino acids) and $\mathrm{C}-(81-206$ amino acids) termini (see Figure 1B), which matched well with the coding domains of GST_N_Sigma_like (CDD accession no.: cd03039) and GST_C_Sigma_like (CDD accession no.: cd03192). In addition, the first ten amino acids corresponded to a signal peptide (Figure 1B), indicating that Bsc-GST $\sigma$ was a secretory GST. Removal of the signal peptide would result in a mature protein with a Mw of $22.398 \mathrm{kDa}$ and a pI of 8.05. Four different Bsc-GST $\sigma$ clones were sequenced in this study, and no differences were found among them at the amino acid level.

Multiple sequence alignment revealed that at the protein level Bsc-GSTo shared the highest similarity (93.2\%) with a GST $\sigma$ from A. suum (GenBank accession no: P46436), followed by three GSTos from $N$. americanus (GenBank accession nos.: ACX53261-ACX53263, mean similarity of $60.4 \%$ ) and GST $\sigma / G S T s$ from two parasitic nematodes Ancylostoma caninum (GenBank accession no.: AAT37718) and Haemonchus contortus (GenBank accession no.: Q9NAW7) as well as a free-living nematode Caenorhabditis elegans (GenBank accession no.: P91253) (41.3-58.7\%; Figure 2). Bsc-GSTo however showed a very

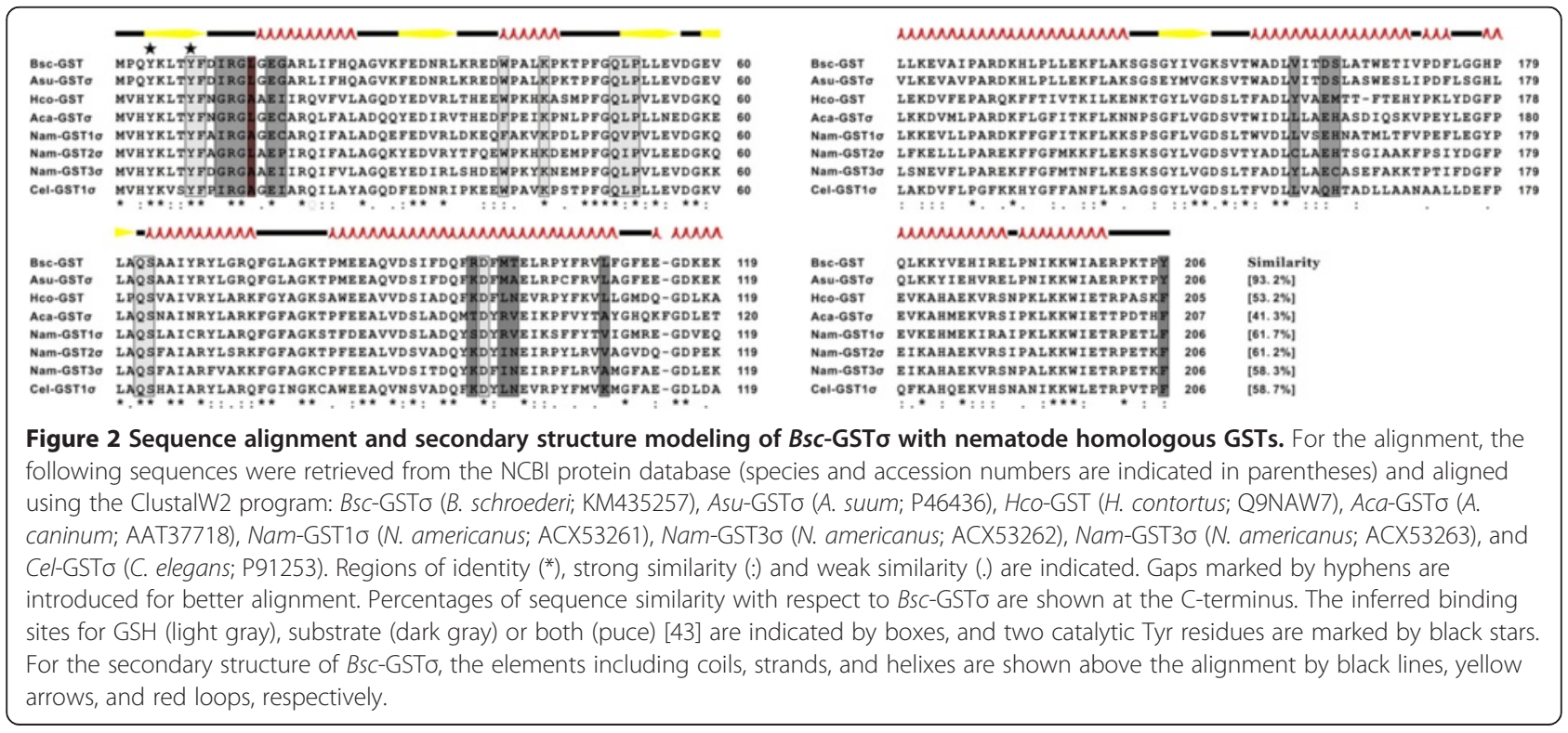


low sequence similarity to GSTs from other organisms including flatworms (e.g., trematodes), insects (e.g., fruitflies), and other mammals (e.g., pandas and swine; data not shown). Further, the amino acid-based alignment demonstrated that the $\mathrm{N}$-terminal region appeared to be highly conserved while the $\mathrm{C}$-terminal region was diverse. For instance, several identical GSH-binding moieties (Tyr-8, Phe-9, Tpr-39, Lys-43, Pro-52, Gln-63, and Asp-97) in the $\mathrm{N}$-terminal domain across all examined lineages were also present in the aforementioned nematode species and occurred without any substitutions in B. schroederi (Figure 2). More importantly, two tyrosine residues associated with the stabilization of GSH [14] were found to be conserved here (Tyr-4 and Tyr-8), including those in Bsc-GST $\sigma$. Conversely, extensive variation was observed at the putative substrate binding pocket ( $\mathrm{H}$-site) located at the $\mathrm{C}$-terminal domain, consistent with the diversified substrate specificities of members of the enzyme family toward xenobiotics. The corresponding substrate-binding moieties in Bsc-GST $\sigma$ were inferred and included: Ile-11, Arg-12, Gly-13, Glu-16, Gly-17, Arg-96, Met-99, Thr-100, Leu109, Val-159, Asp-162, Ser-163, and Tyr-206 (dark gray boxes in Figure 2).

\section{Annotation of Bsc-GSTo structural model}

Similar to other homologous GSTs, the secondary structure of the Bsc-GST $\sigma$ protein consisted of a conserved $\beta \alpha \beta \alpha \beta \beta \alpha$ motif in the $\mathrm{N}$ terminus (ranging from 3 to 74 residues) and a near complete $\alpha$ helix motif (ranging from 81 to 200 residues) in the C-terminus (Figure 2). Based on this secondary structure and the X-ray structure of $N$. americanus GST 62 (PDB no.: 2ON5), the 3D structure of Bsc-GST $\sigma$ was established and shown in Figure 3A. Interestingly, a G-site (GSH interactive region) built by the $\beta \alpha \beta \alpha \beta \beta \alpha$ motif and an $\mathrm{H}$-site built by the near complete $\alpha$ helix motif were determined in the $\mathrm{N}$ - and C-terminal domains, respectively, which was consistent with that of other nematode GSTs characterized to date, including A suum (Figure 3B) [37] and C. elegans (PDB accession no.: 1ZL9; Figure 3C) [38].

\section{Phylogenetic characterization of Bsc-GSTo}

To probe the evolutionary position of $B s c-G S T \sigma$, the amino acid sequences of 64 GSTs included here were aligned and subjected to phylogenetic analysis (Figure 4). The constructed NJ tree clearly supported three major groups: Group 1 (GST $\sigma)$, Group $2(\mathrm{GST} \alpha+\mathrm{GST} \pi+\mathrm{GST} \mu)$, and Group 3 (GST $\theta+$ GST $\xi+$ GST $\Omega+$ GST-Micro). Obviously, Group 3 maintained a greater genetic distance than that between the other two groups, with considerable statistical support (55\%). Among Group 3 and particular Group 2, there were many small clusters formed by different GST classes (e.g., $\alpha, \pi, \mu, \theta, \xi, \Omega$, and Micro) with strong nodal supports (all bootstrap values $\geq 83 \%$ or $=100 \%$ ). For Group 1, the two helminth subgroups (nematodes and trematodes) formed distinct branches with strong supports $(80 \%$ and $87 \%$, respectively). In contrast with the invertebrate helminths, vertebrates formed another independent subgroup with a strong support (81\%) within Group 1. Interestingly, the $B s c-G S T \sigma$ in the nematode-specific subgroup grouped with the GST $\sigma$ of A. suum (100\%) and then with homologues of three strongylid worms $(84 \%)$ and C. elegans ( $\geq 80 \%$; see Figure 4 ).

\section{Expression, purification, and reactivity of $r B s c-G S T \sigma$}

The cDNA encoding mature Bsc-GST $\sigma$ was successfully sub-cloned into the pET32a $(+)$ expression vector (Invitrogen) and expressed in E. coli BL21 (DE3) cells as a single His 6-tagged fusion protein, with an expected size of $\sim 42 \mathrm{kDa}$ (lane 1, Figure 5). Due to an additional $20-\mathrm{kDa}$ epitope tag fusion peptide, the molecular mass of $\mathrm{r} B s c-\mathrm{GST} \sigma$ was $\sim 22 \mathrm{kDa}$, similar to that predicted from its amino acid sequence. Peak expression levels of $\mathrm{r} B s c-\mathrm{GST} \sigma$ occurred at $5 \mathrm{~h}$ induction with IPTG and occurred mostly in inclusion bodies. The $\mathrm{r} B s c$-GST $\sigma$ was purified using a single-step Ni-NTA affinity chromatography under
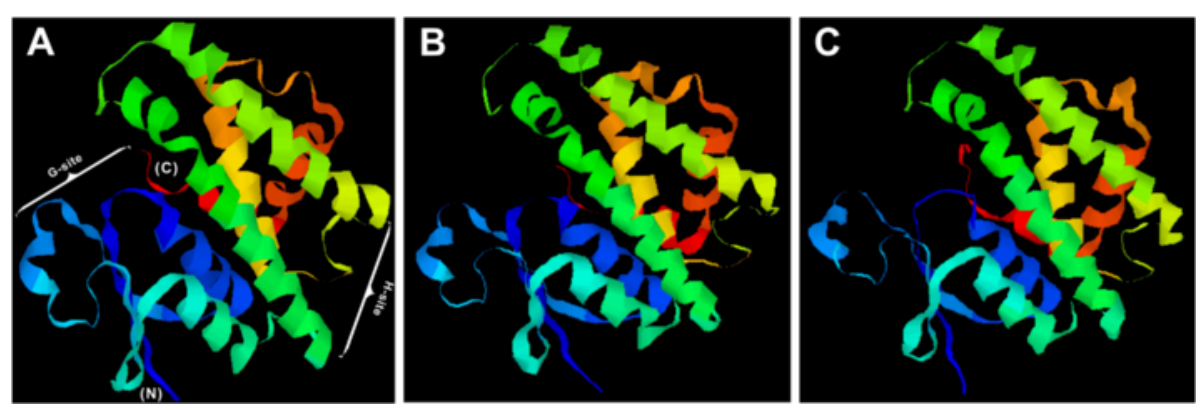

Figure 3 3D models of (A) Bsc-GSTo and two homologous proteins from (B) A. suum and (C) C. elegans. The 3D structures were modeled by referring to the crystal structure of N. americanus GSTб2 (PDB accession no.: 2ON5) using the CPHmodels-3.2 Server program (http://www.cbs. dtu.dk/services/CPHmodels/). N-terminal domain ( $\beta a \beta a \beta \beta a$ motif) constituting G-site and C-terminal domain (a helix motif) constituting H-site are shown in (A) 


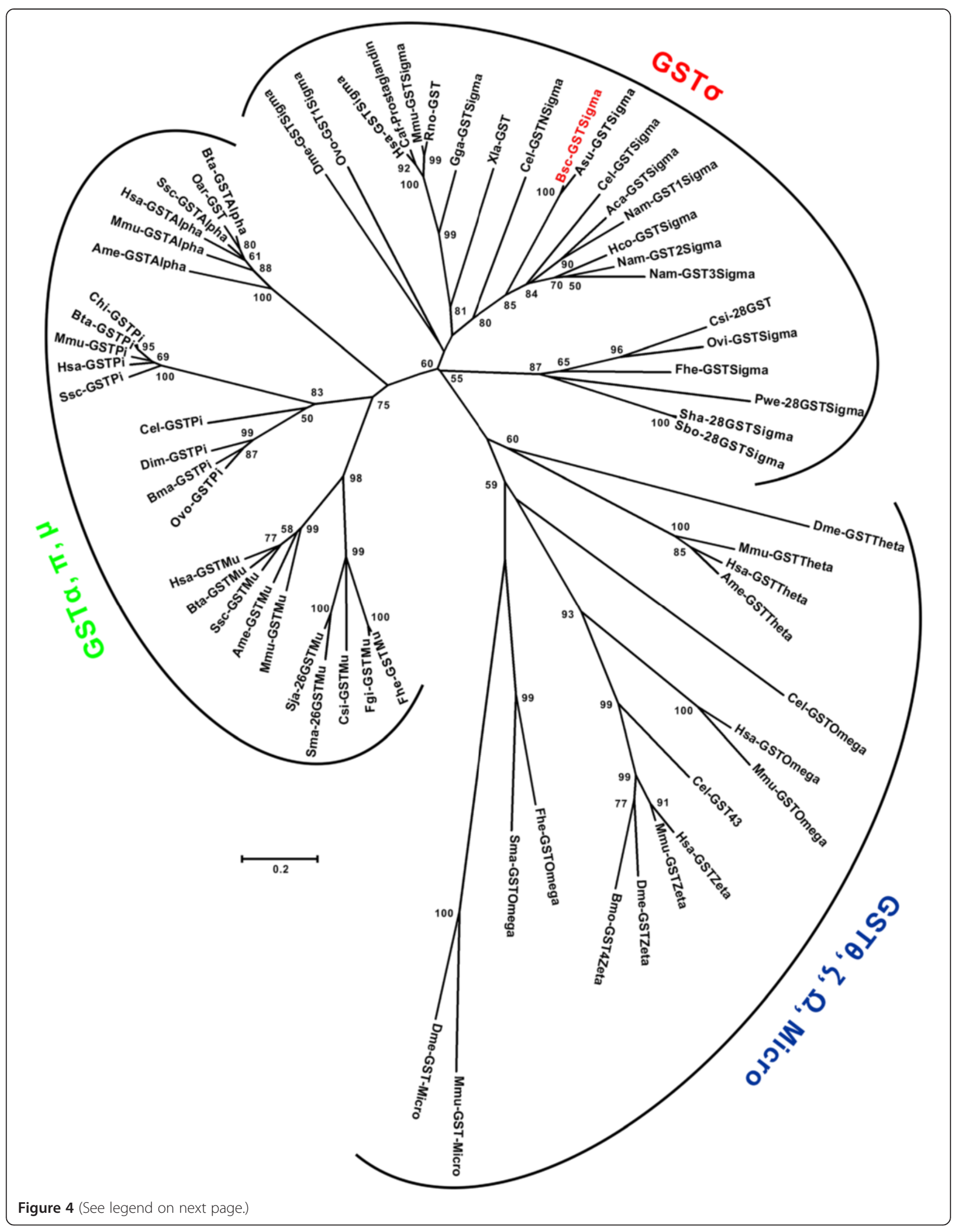




\section{(See figure on previous page.)}

Figure 4 Phylogenetic relationships of $B$. schroederi Bsc-GST $\sigma$ and other classes of GSTs. An unrooted phylogenetic tree was inferred by neighbor-joining (NJ) analysis. Representative GSTs from eight classes: $a, \sigma, \Omega, \theta, \xi, \mu, \pi$, and Micro. The tree was constructed from a multiple sequence alignment performed using ClustalW2 and plotted using MEGA 5.0. Based on the GTR + I + G model, 1,000 bootstrap replicates were run to calculate the percentage reliability for each node, and only values $\geq 50 \%$ are shown. The scale indicates an estimate of substitutions per site, using the optimized model setting. The protein sequences used in the tree are listed as follows, with their GenBank or SwissProt accession numbers: Bos taurus alpha GST (Bta-GSTAlpha), Q28035; Ovis aries GST (Oar-GST), Q9XS30; Sus scrofa alpha GST (Ssc-GSTAlpha), P51781; Homo sapiens alpha GST (Hsa-GSTAlpha), P08263; Mus musculus alpha GST (Mmu-GSTAlpha), Q6P8Q0; Ailuropoda melanoleuca alpha GST (Ame-GSTAlpha), XP_002923004; Drosophila melanogaster sigma GST (Dme-GSTSigma), P41043; Onchocerca volvulus sigma GST (Ovo-GSTSigma), P46434; H. sapiens sigma GST (Hsa-GSTSigma), O60760; Canis familiaris prostaglandin D synthase (Caf-Prostaglandin), NP_001186973; M. musculus sigma GST (Mmu-GSTSigma), Q9JHF7; Rattus norvegicus GST (Rno-GST),O35543; Gallus gallus sigma GST (Gga-GSTSigma), O73888; Xenopus laevis GST (Xla-GST), AAH53774.1; C. elegans sigma GST N (Cel-GSTNSigma), AAB65417; B. schroederi sigma GST (Bsc-GSTSigma), KM435257; A. suum sigma GST (Asu-GSTSigma), P46436; C. elegans sigma GST (Cel-GSTSigma), P91253; A. caninum sigma GST (Aca-GSTSigma), AAT37718; N. americanus sigma GST1 (Nam-GST1Sigma), ACX53261; H. contortus GST (HCo-GST), Q9NAW7; N. americanus sigma GST2 (Nam-GST2Sigma), ACX53262; N. americanus sigma GST3 (Nam-GST3Sigma), ACX53263; Clonorchis sinensis 28-kDa GST (Csi-28GST), O97096; Opisthorchis viverrini sigma GST (Ovi-GSTSigma), AAL23713; Fasciola hepatica sigma GST (Fhe-GSTSigma), ABI79450; Paragonimus westermani 28-kDa sigma GST (Pwe-28GSTSigma), AAB63382; Schistosoma haematobium 28-kDa sigma GST (Sha-28GSTSigma), AAA29892; Schistosoma bovis 28-kDa sigma GST (Sbo-28GSTSigma), AAA29893; C. elegans omega GST (Cel-GSTOmega), P34345; H. sapiens omega GST (Hsa-GSTOmega), P78417; M. musculus omega GST (Mmu-GSTOmega), O09131; F. hepatica omega GST (Fhe-GSTOmega), AFX98104; Schistosoma mansoni omega GST (Sma-GSTOmega), Q86LC0; D. melanogaster theta GST (Dme-GSTTheta), P20432; M. musculus theta GST (Mmu-GSTTheta), Q91X50; H. sapiens theta GST (Hsa-GSTTheta), P30711; A. melanoleuca theta GST (Ame-GSTTheta), XP_002925113; H. sapiens zeta GST (Hsa-GSTZeta),O43708; M. musculus zeta GST (Mmu-GSTZeta), Q9WVL0; D. melanogaster zeta GST (Dme-GSTZeta), Q9VHD3.1; Bombyx mori zeta GST4 (Bmo-GST4Zeta), ABC79691;C. elegans GST 43 (Cel-GST43), Q9N4H6; H. sapiens mu GST (Hsa-GSTMu), P09488; B. taurus mu GST (Bta-GSTMu), Q9N0V4; S. scrofa mu GST (Ssc-GSTMu), Q000H8; A. melanoleuca mu GST (Ame-GSTMu), XP_002919259; M. musculus mu GST (Mmu-GSTMu), P10649; Schistosoma japonicum 26-kDa mu GST (Sja-26GSTMu), P08515; S. mansoni 26-kDa mu GST (Sma-26GSTMu), P15964; C. sinensis mu GST (CSi-GSTMu), AAB46369; F. gigantica mu GST (Fgi-GSTMu), AAD23997; F. hepatica mu GST (Fhe-GSTMu), AHC02709; Capra hircus pi GST (Chi-GSTPi), Q9TTY8; B. taurus pi GST (Bta-GSTPi), P28801; M. musculus pi GST (Mmu-GSTPi), P19157; H. sapiens pi GST (Hsa-GSTPi), P09211; S. scrofa pi GST (Ssc-GSTPi), P80031; C. elegans pi GST (Cel-GSTPi), P10299; Dirofilaria immitis pi GST (Dim-GSTPi), P46426; Brugia malayi pi GST (Bma-GSTPi), CAA73325; O. volvulus pi GST (Ovo-GSTPi), P46427; D. melanogaster micro GST (Dme-GST-Micro), AAC98692; M. musculus micro GST (Mmu-GST-Micro), NP_064330.

denaturing conditions (containing $8 \mathrm{M}$ urea). After refolding and concentration, the purity and yield $(\sim 6 \mathrm{mg} / \mathrm{L})$ of $\mathrm{r} B s c$-GST $\sigma$ were accessed by SDS-PAGE (Figure 5, lanes 2-4). For western blotting analysis, a positive band of $42 \mathrm{kDa}$ was observed when using both rabbit anti- $B$. schroederi serum (experimental group) and mouse anti$\mathrm{r} B s c-\mathrm{GST} \sigma$ serum (positive control), suggesting a strong

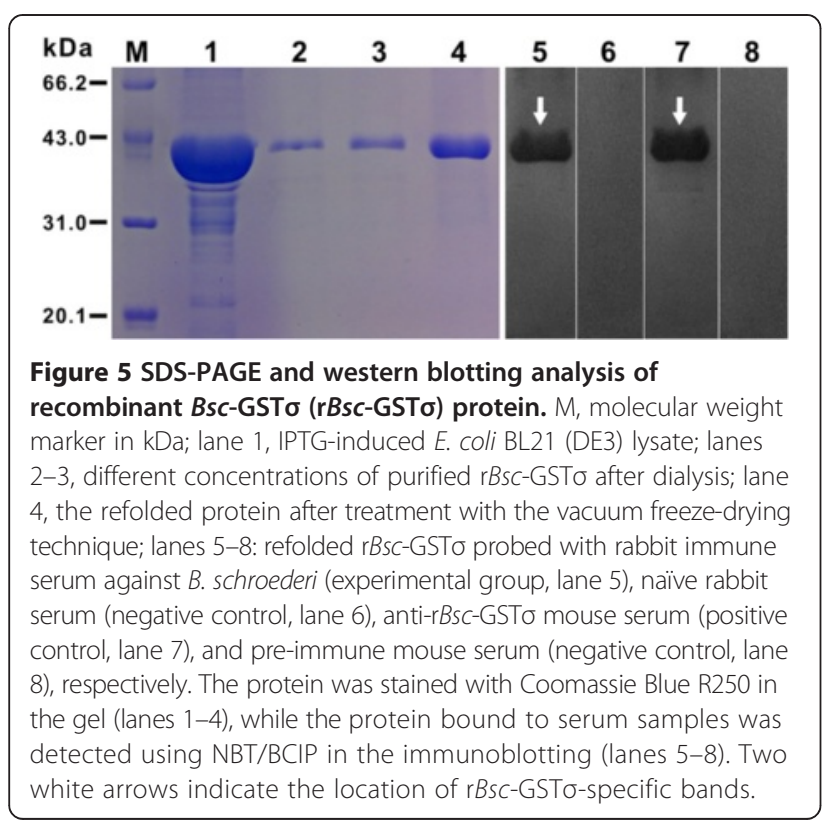

reactivity and good antigenicity of this recombinant protein (Figure 5, lanes 5 and 7). No signal was present in the $\mathrm{r} B s c-\mathrm{GST} \sigma$ incubated with native rabbit and mouse sera (lanes 6 and 8, Figure 5).

\section{Immunolocalization of native Bsc-GST $\sigma$ in adult female B. schroederi}

The tissue distribution of the endogenous Bsc-GST $\sigma$ proteins was located by immunofluorescence assay using anti-rBsc-GST $\sigma$ mouse serum and native mouse serum. Specific fluorescence was clearly observed in sections probed with anti-Bsc-GST $\sigma$ specific serum (Figure 6, panels A-F) but not in those probed with normal mouse serum (Figure 6, panels G-L). The results showed that endogenous Bsc-GST $\sigma$ proteins were mainly localized in several tissues or organs, including the hypodermis, lateral chords, gut epithelium, and gut microvilli of a female adult B. schroederi (panels A-D, Figure 6), which was consistent with its homologous protein in A. suum [36]. Interestingly, we detected strong fluorescence for Bsc-GST $\sigma$ in the ovaries, oviduct epithelium, and egg walls within oviducts, and a lack of fluorescence in muscles and eggs: this was in contrast to its counterparts in A. suum (panels E and F, Figure 6).

\section{Diagnostic potential of rBsc-GSTo protein}

Given the strong antigenicity and reactivity of $\mathrm{r} B s c-\mathrm{GST} \sigma$, an ELISA-based serodiagnostic method was established. 

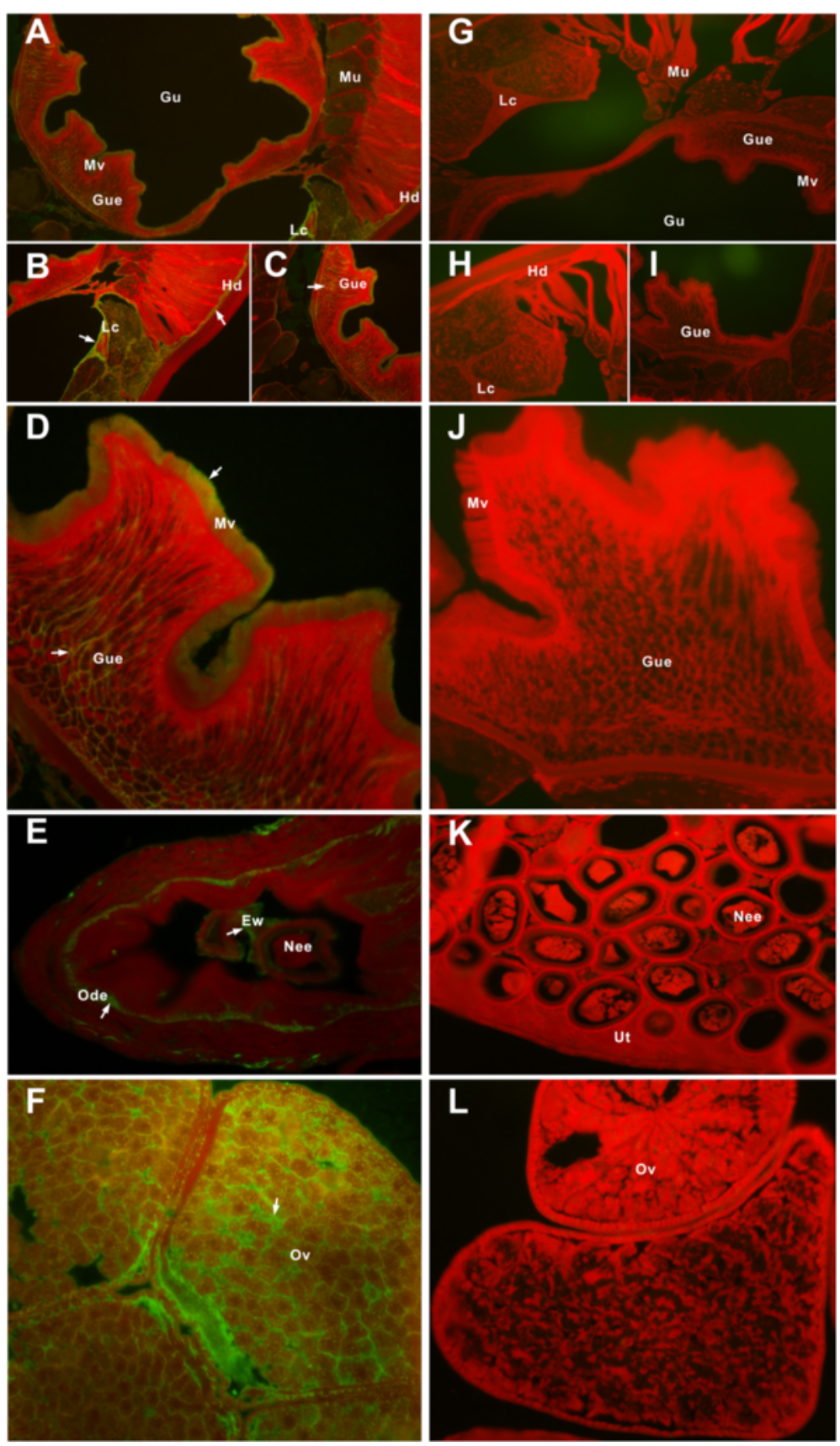

Figure 6 Localization of endogenous Bsc-GSTo in female adult B. schroederi using immunofluorescence. Worms were fixed in paraformaldehyde and embedded in paraffin as described in Methods. The sections (5- $\mu \mathrm{m}$ thickness) were incubated with either mouse anti-rBsc-GSTo serum (1:100; panels A-F) or pre-immune serum at (1:100; panels G-L). The endogenous Bsc-GSTo becomes visible with fluorescein isothiocyanate (FITC)-labeled goat anti-mouse IgG (1:100). White arrows indicate antibody-labeled regions. Different magnifications are used to highlight these regions and

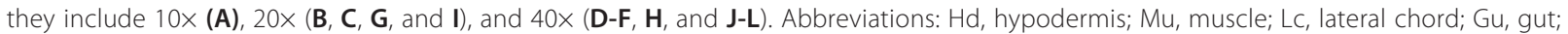
Gue, gut epithelium; Mv, microvilli; Ov, ovary; Ut, uterus; Od, oviduct; Ode, oviduct epithelium; Nee, non-embryonated eggs; Ew, egg wall.

After screening combinations of various amount of antigens tested with various dilutions of positive polyclonal sera, $2 \mu \mathrm{g} / \mathrm{mL}$ of $\mathrm{r} B s c-\mathrm{GST} \sigma$ antigen and 1:800 dilution of sera were deemed optimal for the full set of sample tests. The specific IgG antibodies in all serum samples of mice infected with B. schroederi or with B. transfuga and A. suum were determined (Table 1). Based on the negative cut-off of 0.194 , a total of 34 serum samples from mice infected with $B$. schroederi were detected as positive, corresponding to a sensitivity of $79.1 \%(34 / 43)$. 
Table 1 Detection of anti-B. schroederi IgG antibodies in serum samples of experimentally infected mice ( $\mathrm{N}=$ sample size)

\begin{tabular}{llll}
\hline $\begin{array}{l}\text { Sera of mice infected } \\
\text { with parasites }\end{array}$ & $\mathbf{N}$ & \multicolumn{2}{l}{ rBsc-GSTo-ELISA } \\
\cline { 2 - 4 } & & $\begin{array}{l}\text { OD value } \\
\text { (means } \pm \text { SD) }\end{array}$ & $\begin{array}{l}\text { No. of positive } \\
\text { serum samples (\%) }\end{array}$ \\
\hline B. schroederi & 43 & $1.249 \pm 0.0575$ & $34(79.1)$ \\
B. transfuga & 15 & $0.396 \pm 0.5223$ & $4(26.7)$ \\
A. suum & 15 & $0.432 \pm 0.5118$ & $5(33.3)$ \\
Control & 20 & $0.113 \pm 0.0270$ & $0(0)$ \\
\hline
\end{tabular}

However, due to cross-reactivity with $B$. transfuga-positive mouse sera $(\mathrm{N}=4)$ or $A$. suum-positive mouse sera $(\mathrm{N}=5)$ and no reactions with normal mouse sera, the specificity of the ELISA using $\mathrm{r} B s c$-GST $\sigma$ antigen to detect $B$. schroederi was $82.0 \%(41 / 50)$. Nevertheless, there were statistical differences observed in the ELISA values between the $B$. schroederi-positive sera and the heterogeneous or control sera $(P<0.05$; data not shown). No difference was noted between the heterogeneous and the control sera.

\section{Discussion}

Baylisascariasis, caused by the parasite B. schroederi, is today recognized as a significant cause of mortality of giant pandas, yet the diagnostic tools to detect $B$. schroederi infections in pandas are still lacking [3,39-42]. Numerous studies have highlighted the importance of parasite GSTs, particularly secretory GSTs, in parasite survival and host immune regulation, and thus, as potential candidates for the development of vaccines or diagnostic tools $[15,16,20-22,37]$. With this in mind, in this study we identified and characterized a novel, secretory sigmaclass GST from B. schroederi (Bsc-GST $\sigma$ ) and assessed its potential for serodiagnosis in an experimentally challenged mouse model.

The ongoing annotations of the genome and transcriptome of $B$. schroederi (within our research group) provide a comprehensive bioinformatics platform for probing the accurate complete reference sequence to design primers to amplify and express Bsc-GSTo. By means of the His fusion-based pET32a (+) prokaryotic vector system, a $\sim 42 \mathrm{kDa}$ recombinant Bsc-GST $\sigma$ protein was generated here. Excluding the tag peptides, this $\sim 22-\mathrm{kDa} \mathrm{Mw}$ was well within the range of 21-29 kDa reported for other GSTs [43] and agreed with the findings of a previous review that the average molecular mass of sigma GSTs in parasites is $22 \mathrm{kDa}$ [14]. Further sequencing and structural analyses revealed that Bsc-GST $\sigma$ possessed the typical structural features of representatives of GSTs in the Sigma class: a coding domain for the GST_N_Sigma_like (PSSM: cd03039) and another for the GST_C_Sigma_like (PSSM: cd03192). Meanwhile, the signal peptide analysis implied that $B s c-G S T \sigma$ was a secretory protein. Previous studies have demonstrated that most parasite GSTs isolated to date, including some secretory GSTs, can be grouped independently of their host species into thirteen classes based on their sequence, structure, substrate specificity, and sensitivity to inhibitors (namely alpha, beta, kappa, delta, sigma, theta, mu, omega, pi, tau, zeta, psi, and epsilon) $[13,44,45]$. Combined, the results suggest that BscGST $\sigma$ characterized here should be a secretory GST and is affiliated with the Sigma class.

Sequence alignment and phylogenetic analyses classified Bsc-GSTo along with nematode-specific GSTos, and it together with homologues of other nematodes and trematodes described to date formed a unique, major invertebrate subgroup and were distinct from those of the vertebrates in the Sigma class GSTs (Figure 4), which was consistent with a recently published report [20]. Within the nematode-specific GSTos, eight homologous proteins derived form six parasite species, including $B$. schroederi, were further subjected to pair comparisons on primary, second, and tertiary structure levels. Although Bsc-GSTo was most similar to A. suum GSTo at the amino acid level, the N-terminal seven GSH-binding moieties responsible for the G-site were highly conserved (100\% identity) across all the nematode species included here. Similar conservations were also indicated by their higher (secondary or 3D) structures where a classical GST $\sigma \beta \alpha \beta \alpha \beta \beta \alpha$ motif was present in the N-terminal domain (see Figure 2) [44]. In contrast with G-sit, the $\mathrm{C}$-terminal substrate binding $\mathrm{H}$-site seemed to be greatly variable. Studies confirm that the amino acid identity in the H-site of GSTs is usually very low, even between intraclass members $(<35 \%)$ [46]. This provides the opportunity to screen the region-specific sites for immune responses or to design drugs to inhibit the parasite enzyme only. Furthermore, the distinction of Bsc-GST $\sigma$ from its counterpart in giant pandas further enhances its potential as a diagnostic or therapeutic target due to impossible autoimmune responses caused by cross-reactivity. Under this context, we explored the antigenicity and reactivity of BscGST $\sigma$ using its recombinant form. The results of our immunoblotting analysis showed that $\mathrm{r} B s c-G S T \sigma$ was strongly recognized by rabbit anti- $B$. schroederi serum and mouse anti-rBsc-GST $\sigma$ serum and became visible at $42 \mathrm{kDa}$. These findings confirm the strong immunogenicity of this compound and its potential as a diagnostic tool or candidate target for vaccine development. Two recent studies have successfully used recombinant GST $\sigma$ antigens in vaccines against human hookworm N. americanus (e.g., rNa-GST-1) [47,48] and liver fluke Fasciola hepatica (e.g., rFhGST-S1) [20]. This work suggests that immunoprotective assays to determine the vaccine potential of this recombinant protein in $B$. schroederi are warranted. On the other hand, we 
characterized $B s c$-GST $\sigma$ as a secretory antigen of $B$. schroederi. Previous studies have demonstrated the potential of parasite-specific E/S antigens in diagnostics $[12,49-53]$, thus we thought it prudent to assess the potential of this compound in the serodiagnosis of $B$. schroederi infections in giant pandas. $B$. schroederi infections in wild pandas are not associated with specific clinical symptoms, and the infection can only be confirmed until worms are expelled or eggs are shed in the feces [5]. The development of a serodiagnostic tool will enhance existing diagnostic methods and improve treatment options, especially during the larval migrating stages (L2-L4). After optimizing conditions, a rBsc-GST $\sigma$-based ELISA was established in the present study. Our results revealed that $\mathrm{rBsc}$-GST $\sigma$ could clearly detect the B. schroederi-specific IgG antibodies in experimental mouse sera, with a sensitivity of $79.1 \%$ and a specificity of $82.0 \%$, although cross-reactions were observed in several B. transfuga- or A. suum-positive mouse sera. The presence of cross-reactions may be associated with a similar distribution of epitopes in the GST $\sigma$ homologues of the two ascaridoids that are closely related to B. schroederi. A similar phenomenon was also described between other parasitic nematodes (between Toxocara canis and A. suum and Toxascaris leonina [54], between Anisakis simplex and A. suum and Anisakis physeteris [55], and between Baylisascaris procyonis and Toxocara spp. [56]). The sensitivity of the assay was not high, and is likely a consequence of using a recombinant antigen for a serodiagnostic assay. Recombinant antigens are often produced as single proteins and lack the post-translational chemical or structural modifications present in its native counterpart, which can weaken their sensitivity. Interestingly, it has been shown that combining recombinant antigens can improve the sensitivities of recombinant antigens in the serodiagnosis of parasitic infections [56,57]. Nevertheless, it is important to note that the geographic range of giant pandas does not overlap with that of bears (the hosts for B. transfuga) or swine (the hosts for A. suum). Hence, the chance of cross-reactivity in the wild is low and the serodiagnostic ELISA developed here would be able to detect $B$. schroederi in pandas with a respectable sensitivity.

Finally, our localization analysis showed that native $B s c$-GST $\sigma$ was present in the intestine, reproductive tissues, and body hypodermis of female adult $B$. schroederi. Within the intestine a strong immunofluorescent signal for Bsc-GST $\sigma$ was observed in the brush border of the microvilli, as has been reported for its homologue AsGST1 in A. suum [37]. Surprisingly, this intestinal location was distinct from its homologue OvGST1 in the filaria O. volvulus: the endogenous OvGST1 was distributed in the hypodermis [58]. This discrepancy may be associated with species-specific differences amongst nematodes. With respect to filarial nematodes, they are being considered to become specialized and adopt a blood-based or lymph-based nutrition parasitism, especially their intestines [59]. As compensation for the degeneration or loss of intestinal function, the presence of OvGST1 in the hypodermis of O. volvulus is probably responsible for the metabolism of extrinsic materials and the excretion or secretion of molecules from the parasite, similar to that which occurs in the intestines $[14,15]$. This finding suggests that Bsc-GST $\sigma$ in B. schroederi and AsGST1 in A. suum may both be involved in metabolism and detoxification as well as nutrition during the development of ascaridoids. In addition, we also observed strong fluorescent signals of Bsc-GST $\sigma$ in the ovaries, oviduct epithelium and egg walls, but not in the muscles or ooplasm of B. schroederi. This contrasts to the results reported for their counterparts in A. suum [37]. Whether the expression of this gene in these tissues is also nematode species-specific is not known, but the expression of GSTs in trematode parasites has been reported to be tissue-specific and developmentally regulated. For example, Sm26GST and Sm28GST in Schistosoma mansoni were located in the parenchymal cells of adults but were absent from the musculature, gut and reproductive tissues [60,61]. In F. hepatica, a more recent study showed that FhGST-S1 was present in the parasite tegument, parenchyma, musculature and eggs, and was also found in the excretory/secretory fraction of adults. Thus, the authors inferred that the FhGST-S1 of the adult fluke may play a role in parasite development and the interaction between host and parasite [20]. Perhaps, with the advent of readily available genomic tools, the combination of high-throughput genome sequencing and mass spectrometry techniques for exploring pannematode proteomics will provide a comprehensive platform for an in-depth structure and function analyses of these GSTos. This should contribute to a better understanding of the biological roles of nematode-specific GSTos.

\section{Conclusions}

The full-length cDNA encoding a novel Sigma class glutathione S-transferases (Bsc-GST $\sigma$ ) from B. schroederi was identified by screening the genome and trancriptome datasets and subsequently cloned and expressed. We determined some of the structural characteristics and tissue-specific distributions of this compound, providing insights into the biological functions of this protein. Furthermore, we showed that $\mathrm{rBsc}$-GST $\sigma$ has strong immunogenicity and we confirmed via a serodiagnostic assay that $\mathrm{rBsc}$-GST $\sigma$ is a suitable diagnostic antigen and could be used to develop an ELISA-based serological test for the diagnosis, seroepidemiology, and serosurveillance of $B$. schroederi in giant pandas. 


\section{Competing interests}

The authors declare that they have no competing interests.

\section{Authors' contributions}

$X Y$ and $Z X$ participated in the design of the study, performed all experiments, collected and analyzed data, and completed manuscript preparation. WT, GXB, ZZH, WCD and PXR participated in collection of parasite specimens and carried out the mouse infection experiments. WT and $\mathrm{CL}$ contributed with the design and analyses of serodiagnostic trials. YGY conceived of the study, participated in its design and coordination, and helped to interpret the results and edited the manuscript. All authors read and approved the final manuscript.

\section{Acknowledgements}

This work was supported by the grant from the Research Fund for the Chengdu Giant Panda Breeding (No. CPF-2012-13). We would like to thank Mei Liu and Yu Wang (College of Veterinary Medicine, Sichuan Agricultural University, China) for technical assistance; Caiwu Li (Ya'an Bifengxia Research Base of Giant Panda Breeding, China) for materials.

\section{Author details}

${ }^{1}$ Department of Parasitology, College of Veterinary Medicine, Sichuan Agricultural University, Ya'an 625014, China. ${ }^{2}$ Centre for Animal Diseases Control and Prevention, Dachuan Animal Husbandry Bureau, Dazhou 623000, China. ${ }^{3}$ Chengdu Research Base of Giant Panda Breeding, Chengdu 610081, China. ${ }^{4}$ China Conservation and Research Center for Giant Panda, Wolong 623006, China. ${ }^{5}$ Department of Chemistry, College of Life and Basic Science, Sichuan Agricultural University, Ya'an 625014, China.

\section{Received: 15 December 2014 Accepted: 29 December 2014} Published online: 23 January 2015

\section{References}

1. Wei F, Hu Y, Zhu L, Bruford MW, Zhan X, Zhang L. Black and white and read all over: the past, present and future of giant panda genetics. Mol Ecol. 2012;21:5660-74

2. Yang B, Busch J, Zhang L, Ran J, Gu X, Zhang W, et al. Eco-compensation for giant panda habitat. Science. 2013;339:521.

3. Zhang JS, Daszak P, Huang HL, Yang GY, Kilpatrick AM, Zhang S. Parasite threat to panda conservation. Ecohealth. 2008;5:6-9.

4. Wildt DE, Zhang AJ, Zhang HM, Janssen DL, Ellis S. Giant Pandas: Biology, Veterinary Medicine and Management. Cambridge: Cambridge University Press; 2006.

5. Loeffler K, Montali RJ, Rideout BA. Diseases and pathology of giant pandas. In: Wildt DE, Zhang ZA, Zhang HM, Janssen DL, Ellis S, editors. Giant Pandas: Biology, Veterinary Medicine and Management, Cambridge. Cambridge: Cambridge University Press; 2006. p. 377-409.

6. Wang T, He G, Yang G, Fei Y, Zhang Z, Wang C, et al. Cloning, expression and evaluation of the efficacy of a recombinant Baylisascaris schroederi Bs-Ag3 antigen in mice. Vaccine. 2008;26:6919-24.

7. Lin Q, Li H, Gao M, Wang X, Ren W, Cong M, et al. Characterization of Baylisascaris schroederi from Qinling subspecies of giant panda in China by the first internal transcribed spacer (ITS-1) of nuclear ribosomal DNA Parasitol Res. 2012;110:1297-303.

8. Xie Y, Chen S, Yan Y, Zhang Z, Li D, Yu H, et al. Potential of recombinant inorganic pyrophosphatase antigen as a new vaccine candidate against Baylisascaris schroederi in mice. Vet Res. 2013;44:90.

9. Zhou X, Yu H, Wang N, Xie Y, Liang Y, Li D, et al. Molecular diagnosis of Baylisascaris schroederi infections in giant panda (Ailuropoda melanoleuca) feces using PCR. J Wildl Dis. 2013;49:1052-5.

10. Zhang W, Yie S, Yue B, Zhou J, An R, Yang J, et al. Determination of Baylisascaris schroederi infection in wild giant pandas by an accurate and sensitive PCR/CE-SSCP method. PLoS One. 2012;7:e41995.

11. Wang N, Li D, Zhou X, Xie Y, Liang Y, Wang C, et al. A sensitive and specific PCR assay for the detection of Baylisascaris schroederi eggs in giant panda feces. Parasitol Int. 2013;62:435-6.

12. Ranganathan S, Garg G. Secretome: clues into pathogen infection and clinical applications. Genome Med. 2009;1:113.

13. Salinas AE, Wong MG. Glutathione S-transferases-a review. Curr Med Chem. 1999;6:279-310
14. Torres-Rivera A, Landa A. Glutathione transferases from parasites: a biochemical view. Acta Trop. 2008;105:99-112.

15. Brophy $P$, Barrett J. Glutathione transferase in helminths. Parasitology. 1990;100:345-9.

16. Cervi L, Rossi G, Masih DT. Potential role for excretory-secretory forms of glutathione-S-transferase (GST) in Fasciola hepatica. Parasitology. 1999;119:627-33.

17. Guillou F, Roger E, Moné Y, Rognon A, Grunau C, Théron A, et al. Excretorysecretory proteome of larval Schistosoma mansoni and Echinostoma caproni, two parasites of Biomphalaria glabrata. Mol Biochem Parasitol. 2007;155:45-56.

18. Brophy P, Patterson L, Brown A, Pritchard D. Glutathione S-transferase (GST) expression in the human hookworm Necator americanus: potential roles for excretory-secretory forms of GST. Acta Trop. 1995;59:259-63.

19. Liebau E, Höppner J, Mühlmeister M, Burmeister C, Lüersen K, Perbandt M, et al. The secretory omega-class glutathione transferase OvGST3 from the human pathogenic parasite Onchocerca volvulus. FEBS J. 2008;275:3438-53.

20. LaCourse EJ, Perally S, Morphew RM, Moxon JV, Prescott M, Dowling DJ, et al. The Sigma class glutathione transferase from the liver fluke Fasciola hepatica. PLoS Negl Trop Dis. 2012;6:e1666.

21. Kampkötter A, Volkmann TE, de Castro SH, Leiers B, Klotz LO, Johnson TE, et al. Functional analysis of the glutathione S-transferase 3 from Onchocerca volvulus (Ov-GST-3): a parasite GST confers increased resistance to oxidative stress in Caenorhabditis elegans. J Mol Biol. 2003;325:25-37.

22. Zhan B, Perally S, Brophy PM, Xue J, Goud G, Liu S, et al. Molecular cloning, biochemical characterization, and partial protective immunity of the heme-binding glutathione S-transferases from the human hookworm Necator americanus. Infect Immun. 2010;78:1552-63.

23. Brophy PM, Krause S, Walter RD, Liebau E, Fischer P, Sommer A. Gene structure of the extracellular glutathione S-transferase from Onchocerca volvulus and its overexpression and promoter analysis in transgenic Caenorhabditis elegans. Mol Biochem Parasitol. 2001;117:145-54.

24. Acevedo N, Mohr J, Zakzuk J, Samonig M, Briza P, Erler A, et al. Proteomic and immunochemical characterization of glutathione transferase as a new allergen of the nematode Ascaris lumbricoides. PLoS One. 2013;8:e78353.

25. Crompton D. Ascaris and ascariasis. Adv Parasitol. 2001;48:285-375.

26. Bowman DD, Little MD, Reimers RS. Precision and accuracy of an assay for detecting Ascaris eggs in various biosolid matrices. Water Res. 2003;37:2063-72.

27. Unites States Environmental Protection Agency (USEPA). Environmental regulations and technology: Control of pathogens and vectorattractionin sewage sludge. Ohio: EPA/625/R-92/013; 2003.

28. Thompson JD, Higgins DG, Gibson TJ. CLUSTAL W: improving the sensitivity of progressive multiple sequence alignment through sequence weighting, position-specific gap penalties and weight matrix choice. Nucleic Acids Res. 1994;22:4673-80

29. Tamura K, Peterson D, Peterson N, Stecher G, Nei M, Kumar S. MEGA5: molecular evolutionary genetics analysis using maximum likelihood, evolutionary distance, and maximum parsimony methods. Mol Biol Evol. 2011;28:2731-9.

30. Bell BA, Wood JF, Bansal R, Ragab H, Cargo III J, Washington MA, et al. Process development for the production of an E. coli produced clinical grade recombinant malaria vaccine for Plasmodium vivax. Vaccine. 2009;27:1448-53

31. Tsuji N, Suzuki K, Kasuga-Aoki H, Isobe T, Arakawa T, Matsumoto Y. Mice intranasally immunized with a recombinant 16-kilodalton antigen from roundworm Ascaris parasites are protected against larval migration of Ascaris suum. Infect Immun. 2003;71:5314-23.

32. Fu Y, Nie HM, Niu LL, Xie Y, Deng JB, Wang Q, et al. Comparative efficacy of ivermectin and levamisole for reduction of migrating and encapsulated larvae of Baylisascaris transfuga in mice. Korean J Parasitol. 2011;49:145-51.

33. Cui J, Wei T, Liu LN, Zhang X, Qi X, Zhang ZF, et al. Molecular characterization of a Spirometra mansoni antigenic polypeptide gene encoding a 28.7 kDa protein. Parasitol Res. 2014;113:3511-6.

34. Vlaminck J, Nejsum P, Vangroenweghe F, Thamsborg SM, Vercruysse J, Geldhof P. Evaluation of a serodiagnostic test using Ascaris suum haemoglobin for the detection of roundworm infections in pig populations. Vet Parasitol. 2012;189:267-73.

35. Erlwein O, Robinson MJ, Kaye S, Wills G, Izui S, Wessely S, et al. Investigation into the presence of and serological response to XMRV in CFS patients. PLoS One. 2011;6:e17592.

36. Breathnach R, Chambon P. Organization and expression of eucaryotic split genes coding for proteins. Annu Rev Biochem. 1981;50:349-83. 
37. Liebau E, Eckelt VH, Wildenburg G, Teesdale-Spittle P, Brophy PM, Walter RD, et al. Structural and functional analysis of a glutathione S-transferase from Ascaris suum. Biochem J. 1997;324:659-66.

38. Babu RO, Moorkoth D, Azeez S, Eapen SJ. Virtual screening and in vitro assay of potential drug like inhibitors from spices against Glutathione-STransferase of Meloidogyne incognita. Bioinformation. 2012;8:319-25.

39. Zhang L, Yang X, Wu H, Gu X, Hu Y, Wei F. The parasites of giant pandas: individual-based measurement in wild animals. J Wildl Dis. 2011;47:164-71.

40. Xie Y, Zhang Z, Wang C, Lan J, Li Y, Chen Z, et al. Complete mitochondrial genomes of Baylisascaris schroederi, Baylisascaris ailuri and Baylisascaris transfuga from giant panda, red panda and polar bear. Gene. 2011;482:59-67.

41. Zhao GH, Xu MJ, Zhu XQ. Identification and characterization of microRNAs in Baylisascaris schroederi of the giant panda. Parasit Vectors. 2013;6:216.

42. Yang GY, Zhang ZH. Parasitic diseases of wild life. Beijing: Science Press; 2013.

43. Mannervik B, Danielson UH. Glutathione transferases-structure and catalytic activity. CRC Crit Rev Biochem. 1988;23:283-337.

44. Umasuthan N, Saranya Revathy K, Lee Y, Whang I, Choi CY, Lee J. A novel molluscan sigma-like glutathione S-transferase from Manila clam, Ruditapes philippinarum: cloning, characterization and transcriptional profiling. Comp Biochem Physiol C Toxicol Pharmacol. 2012;155:539-50

45. Lee AJ, Huntley J, Van den Broek A, Coates D, Isaac RE. Expression and characterisation of a Psoroptes ovis glutathione S-transferase. Vet Parasitol. 2002;105:49-63.

46. Blanchette B, Feng X, Singh BR. Marine glutathione S-transferases. Mar Biotechnol. 2007:9:513-42.

47. Hotez PJ, Diemert D, Bacon KM, Beaumier C, Bethony JM, Bottazzi ME, et al. The human hookworm vaccine. Vaccine. 2013:31:B227-32.

48. Curti E, Seid CA, Hudspeth E, Center L, Rezende W, Pollet J, et al. Optimization and revisions of the production process of the Necator americanus glutathione S-transferase 1 (Na-GST-1), the lead hookworm vaccine recombinant protein candidate. Hum Vaccin Immunother. 2014;10:1914-25

49. Kennedy MW, Qureshi F. Stage-specific secreted antigens of the parasitic larval stages of the nematode Ascaris. Immunology. 1986;58:515-22.

50. Odoevskaia IM. Diagnostic efficiency of excretory-secretory Trichinella antigens in enzyme immunoassay. Med Parazitol (Mosk). 2007:4:19-24. in Russian.

51. Watthanakulpanich D, Smith HV, Hobbs G, Whalley AJ, Billington D. Application of Toxocara canis excretory-secretory antigens and lgG subclass antibodies (lgG1-4) in serodiagnostic assays of human toxocariasis. Acta Trop. 2008;106:90-5.

52. Gómez-Morales MA, Ludovisi A, Amati M, Pozio E. Validation of an excretory/secretory antigen based-ELISA for the diagnosis of Opisthorchis felineus infection in humans from low trematode endemic areas. PLoS One. 2013;8:e62267.

53. Burk SV, Dangoudoubiyam S, Brewster-Barnes T, Bryant UK, Howe DK, Carter CN, et al. In vitro culture of Parascaris equorum larvae and initial investigation of parasite excretory-secretory products. Parasitol Res. 2014;113:4217-24.

54. Cuéllar C, Fenoy S, Guillén JL. Cross-reactions of sera from Toxocara canis-infected mice with Toxascaris leonina and Ascaris suum antigens. Int J Parasitol. 1992;22:301-7.

55. Lozano MJ, Martín HL, Díaz SV, Mañas Al, Valero LA, Campos BM. Cross-reactivity between antigens of Anisakis simplex s.l. and other ascarid nematodes. Parasite. 2004;11:219-23.

56. Dangoudoubiyam S, Vemulapalli R, Ndao M, Kazacos KR. Recombinant antigen-based enzyme-linked immunosorbent assay for diagnosis of Baylisascaris procyonis larva migrans. Clin Vaccine Immunol. 2011;18:1650-5.

57. Greve JH, O'Brien SE. Adult Baylisascaris infections in two dogs. Comp Anim Pract. 1989;19:41-3.

58. Liebau E, Wildenburg G, Walter RD, Henkle-Dührsen K. A novel type of glutathione S-transferase in Onchocerca volvulus. Infect Immun. 1994;62:4762-7.

59. Striebel HP. Proposed form to evaluate some histological aspects of macrofilarial morphology, its age dependent alterations and drug related changes in nodules of Onchocerca volvulus and O. gibsoni. Trop Med Parasitol. 1988:39:367-89.

60. Porchet E, McNair A, Caron A, Kusnierz JP, Zemzoumi K, Capron A. Tissue expression of the Schistosoma mansoni 28 kDa glutathione S-transferase. Parasitology. 1994;109:565-72.
61. Trottein F, Kieny MP, Verwaerde C, Torpier G, Pierce RJ, Balloul JM, et al. Molecular cloning and tissue distribution of a 26-kilodalton Schistosoma mansoni glutathione S-transferase. Mol Biochem Parasitol. 1990;41:35-44.

\section{Submit your next manuscript to BioMed Central and take full advantage of:}

- Convenient online submission

- Thorough peer review

- No space constraints or color figure charges

- Immediate publication on acceptance

- Inclusion in PubMed, CAS, Scopus and Google Scholar

- Research which is freely available for redistribution 